\title{
We can have it all, but we just cannot have it all at once
}

\author{
Yoshihiko Kanno ${ }^{1}$
}

Received: 7 March 2020 / Accepted: 11 March 2020 / Published online: 2 April 2020

(c) The Japanese Society of Hypertension 2020

Although dialysis therapy in Japan has a 60-year history and we have achieved some of the best results in the world, blood pressure management in dialysis patients has remained an endless theme for specialists in the fields of hypertension and dialysis. Blood pressure is one of the most crucial factors affecting the occurrence of cardiovascular events, and many clinicians have encountered this wellknown problem in dialysis patients. So how can we control blood pressure to improve the prognosis of dialysis patients? Despite the fact that blood pressure of nondialysis patients is an evidence-rich area, regarding dialysis patients, it has not even been determined as to what point in time blood pressure should be used as an indicator of cardiovascular events [1]. Although there is no significant difference between healthy subjects and dialysis patients regarding the cause of hypertension, the disruption of renal function results in a certain degree of the loss of blood pressure control. The acquired causes of the loss of blood pressure control include (1) body fluid excess, (2) abnormalities of the renin-angiotensin system, (3) enhancement of sympathetic activity, (4) disorders of endothelial-dependent vasodilation, and (5) side effects of erythropoietin treatment. Particularly in anuric patients, fluid excess substantially affects blood pressure, and hence, the maximum value before dialysis at the beginning of the week is robustly increased. Blood pressure is reduced when water removal by dialysis is performed, but when the water removal rate is high, it may lead to shock-level hypotension caused by intravascular collapse. Therefore, blood pressure control of dialysis patients has been considered difficult, fluctuating between fluid excess and fluid insufficiency.

In the study published in this issue of Hypertension Research, Tsuruya et al. demonstrated that postdialysis

Yoshihiko Kanno

kannoyh@tokyo-med.ac.jp

1 Department of Nephrology, Tokyo Medical University, 6-7-1 Nishishinjuku, Shinjuku, Tokyo 160-023, Japan systolic blood pressure and pulse pressure are more accurate predictors of all-cause and cardiovascular mortality than predialysis systolic blood pressure and showed U-shaped associations with these outcomes in 2690 Japanese hemodialysis patients [2]. In their study, both predialysis and postdialysis systolic and diastolic blood pressure, as well as pulse pressure, showed U-shaped hazard ratios for all-cause and cardiovascular mortality. The lowest risk for cardiovascular mortality was found in those with predialysis and postdialysis blood pressures of $120-139 \mathrm{mmHg}$. This value is much lower than those in previous studies, which reported blood pressures of $140-159 \mathrm{mmHg}$ [3]. As the lowest risk of this U-curve in studies from overseas is also at a high blood pressure of $160 \mathrm{mmHg}$ [4], evidence-based decisions will be substantially different from the management goals for nondialysis persons [1]. Even regarding the data from the same Japanese dialysis patients, the results of the primary endpoint may change with the use of different analytical methods and different sample extraction techniques. Of course, the optimal target must be changed to analyze various data in various ways. The novelty of the study by Tsuruya et al. is that it gives significance to postdialysis blood pressure, which has not previously received much attention from researchers and clinicians. The Japanese Society of Dialysis Therapy proposed in their 2010 guidelines to set the predialysis systolic blood pressure at the beginning of the week to $<140 / 90 \mathrm{mmHg}$ [5]. However, the rate of compliance to these guidelines has not been very high because this value did not match those used for management in the clinical field. The reason may be that many patients experience hypotension during the dialysis session. If the predialysis systolic blood pressure is set below $140 \mathrm{mmHg}$ in accordance with these guidelines, many clinicians may worry about the possibility of hypotension occurring during dialysis in some patients, which has been considered a risk factor for patient prognosis, similarly to hypoglycemia [6].

Tsuruya et al. also demonstrated that a postdialysis systolic blood pressure of $120-139 \mathrm{mmHg}$ demonstrated the lowest risk of cardiovascular events. On the basis of these 
results, when the postdialysis systolic blood pressure is set to $120-139 \mathrm{mmHg}$, it may be possible to avoid hypotension. In addition, considering that the most favorable predialysis systolic blood pressure is also $120-139 \mathrm{mmHg}$, patients who are not anuric, patients who have similar predialysis and postdialysis blood pressures, patients who have sufficient cardiac function, and patients with only a small amount of fluid removal at a dialysis session may have a good prognosis. On the other hand, a postdialysis systolic blood pressure of $120-139 \mathrm{mmHg}$ might require some patients to have systolic blood pressures of dangerous levels $(\geq 180 \mathrm{mmHg})$ at the start. Although it is possible to derive various hypotheses from the results of one study, it is difficult to verify them. This is why there has been a decrease in research on blood pressure and dialysis. It has been thought to be difficult to establish an index that is appropriate and that can be readily understood by all staff and patients. However, after several years of consideration, in 2017, a proposal for dialysis patients was presented by the Joint Working Group of the European Society of Kidney/Dialysis Transplantation and the European Society of Hypertension, namely, an average value of $135 / 85 \mathrm{mmHg}$ or less for home blood pressure measured in the morning and evening during 6 nondialysis days or an average blood pressure of below $130 / 80 \mathrm{mmHg}$ measured by $24-\mathrm{h}$ ambulatory monitoring on a nondialysis day during the week [7]. This is considered to be a new viewpoint, although the care of patients who cannot obtain their own blood pressure at home must be considered. Again, this report from Tsuruya et al. demonstrated that postdialysis blood pressure is also a potential indicator. Therefore, although blood pressure control indicators for dialysis patients may be a theme that was once considered to be too difficult, recent research, such as with the use of big data, is providing new clues. If we continue our steady efforts to consider each and every indicator, we should be able to establish a viable one in the near future.

\section{Compliance with ethical standards}

Conflict of interest The author received honoraria and/or fees for the promotional materials from Kyowa Kirin, Chugai Pharmaceutical, Kissei Pharmaceutical, and Fuji Yakuhin and also received research funding from Kyowa Kirin and Chugai Pharmaceutical.

Publisher's note Springer Nature remains neutral with regard to jurisdictional claims in published maps and institutional affiliations.

\section{References}

1. Umemura S, Arima H, Arima S, Asayama K, Dohi Y, Hirooka Y, et al. The Japanese Society of Hypertension guidelines for the management of hypertension (JSH 2019). Hypertension Res. 2019;42:1235-481.

2. Tsuruya K, Kanda E, Nomura T, Iseki K, Hirakata H. Postdialysis blood pressure is a better predictor of mortality than predialysis blood pressure in Japanese hemodialysis patients: the Japan Dialysis Outcomes and Practice Patterns Study. Hypertens Res. 2020. https://doi.org/10.1038/s41440-020-0425-1.

3. Inaba M, Karaboyas A, Akiba T, Akizawa T, Saito A, Fukuhara S, et al. Association of blood pressure with all-cause mortality and stroke in Japanese hemodialysis patients: the Japan Dialysis Outcomes and Practice Pattern Study. Hemodial Int. 2014;18:607-15.

4. Hannedouche $T$, Roth $H$, Krummel T, London GM, Jean G, Bouchet JL, et al. Multiphasic effects of blood pressure on survival in hemodialysis patients. Kidney Int. 2016;90:674-84.

5. Hirakata H, Nitta K, Inaba M, Shoji T, Fujii H, Kobayashi S, et al. Japanese Society for Dialysis Therapy guidelines for management of cardiovascular diseases in patients on chronic hemodialysis. Therapeutic Apheresis Dialysis. 2012;16:387-435.

6. Shoji T, Tsubakihara Y, Fujii M, Imai E. Hemodialysis-associated hypotension as an independent risk factor for two-year mortality in hemodialysis patients. Kidney Int. 2004;66:1212-20.

7. Sarafidis PA, Persu A, Agarwal R, Burnier M, de Leeuw P, Ferro CJ, et al. Hypertension in dialysis patients: a consensus document by the European Renal and Cardiovascular Medicine (EURECA-m) working group of the European Renal AssociationEuropean Dialysis and Transplant Association (ERA-EDTA) and the Hypertension and the Kidney working group of the European Society of Hypertension (ESH). Nephrol Dial Transplant. 2017;32:620-40. 